\title{
Pathological Diagnosis: The Need of the Hour
}

\author{
Nithya Jagannathan ${ }^{1^{*}}$ and Pratibha Ramani ${ }^{2}$ \\ ${ }^{1}$ Senior Lecturer, Department of Oral Pathology, Saveetha Dental College, Chennai, India \\ ${ }^{2}$ Professor and Head of the Department, Department of Oral Pathology, Saveetha Dental College, Chennai, India
}

*Corresponding author: Dr. Nithya Jagannathan, Department of Oral Pathology, Saveetha Dental College, No 162, Poonamalle High Road, Vellapanchavadi, Chennai, India 600 077, Tel: +9884754910; E-mail: dr.nithya@ymail.com

Rec date: Jun 28, 2014, Acc date: Jun 28, 2014, Pub date: Jun 30, 2014

Copyright: (c) 2014 Jagannathan N, et al. This is an open-access article distributed under the terms of the Creative Commons Attribution License, which permits unrestricted use, distribution, and reproduction in any medium, provided the original author and source are credited

\section{Editorial}

Diagnostic accuracy is the most essential aspect of medical and dental practice, nevertheless, this also appears to be the most challenging aspect. The role of pathologists in the process of diagnosis cannot be over emphasized. Diagnosis is like a puzzle, with each piece contributing to some detail. Perfect assembly of all the information into a single picture reveals the mystery of the disease process. It is often said "our eyes see what the mind perceives". Pathology is an art that requires certain level of intellect and imagination to make a diagnosis. Pathology is an evolving specialty which has shown considerable growth in the medical and dental fields. Pathology, once a discipline included under clinical practice has emerged into several branches including molecular biology, genetics, immunology and forensics which have led to its recognition as a unique branch by itself. The high quality clinical care and optimum treatment needs in all areas of clinical essentials is based on microscopic interpretation of a lesion. Pathology hence forms a mainstay not just a diagnostic aid, but also in rendering excellence in clinical areas [1].

McLendon aptly defined a pathologist as a physician who understands the disease process and puts in considerable efforts to eliminate the disease process, a scientist with an inquiring intellect probing into all aspects of research, a facilitator sharing the clinical acumen and knowledge with the students and colleagues and a leader as pathology serves as a major role in advancement of medicine [2].

Pathology is often integrated with clinical informatics to arrive at an accurate diagnosis. It was quoted by George Bernard Shaw that "sending a specimen to a pathologist without clinical information is a subtle tribute to his omniscience, but diagnostically worthless" [3]. The statement is applicable in almost all the cases. In the present days, pathology is incorporated into clinical practice through a multidisciplinary team which increases the standard of patient care. Histopathology involves addressing of a specimen visually, anatomically and microscopically. The pathologist starts his diagnosis when he visualizes the specimen and observes the consistency and other macroscopic changes during the grossing stages of the specimen $[4,5]$. Progress in pathological diagnosis has made a tremendous advancement in the past decade in terms of molecular advancements and genetics.

In the present day, new techniques have emerged in investigating the disease process which has revolutionized the genetics and biology the disease. The accuracy of diagnosis is further enhanced with sophistication of microscopes and molecular investigations which has expanded pathology from mere morphological interpretation to molecular and genetic analysis. The ability to understand the molecular basis of a disease and its application in histopathological interpretation makes pathology the keystone for diagnosis and management that will characterize the field of medicine and dentistry in the next millennium.

\section{References}

1. Fleming S (2012) Clinical Pathology - A Diagnostic Aid?. Scottish Univ Med J 1: 192-198

2. Mc Lendon WW (1986) A historical perspective as a compass for the future of pathology. Arch Pathol Lab Med 110: 284-289.

3. J. Tei Anim (2007) The changing role of the pathologist. Bull Kuwait Inst Med Spec 6: 8-12

4. Dyke SC (1984) As quoted by Forster WD. Pathology as a profession in Great Britain and the early history of the Royal College of Pathologists. The Royal College of Pathologists.

5. Lambird PA (1986) Pathology and the corporate transformation of Americal Medcine. Arch Pathol Lab Med 110: 275-279. 